\title{
Usefulness of inspiratory capacity measurement in COPD patients in the primary care setting
}

This article was published in the following Dove Press journal:

International Journal of General Medicine

29 October 2009

Number of times this article has been viewed

\author{
Antonio Madueño' \\ Antonio Martín² \\ Juan-Antonio Péculo 3 \\ Esther Antón ${ }^{2}$ \\ Alejandra Paravisini ${ }^{2}$ \\ Antonio León ${ }^{4}$ \\ 'Specialist in Family and Community \\ Care Medicine, Centro de Salud \\ La Laguna, Cádiz, Spain; ${ }^{2}$ Medical \\ Department, Pfizer, Madrid, \\ Spain; ${ }^{3}$ Nurse, Empresa Pública \\ de Emergencias Sanitarias, Cádiz, \\ Spain; ${ }^{4}$ Section Chief of Pneumology, \\ Puerta del Mar University Hospital, \\ Cádiz, Spain
}

Objective: To determine if inspiratory capacity (IC) assessment could be useful for chronic obstructive pulmonary disease (COPD) patient management in the primary care setting.

Methods: A descriptive cross-sectional study was conducted in 93 patients diagnosed with COPD according to Spanish Thoracic Society (SEPAR) criteria. Patients were recruited in eight primary care centers in Andalusia, Spain. Anthropometric, sociodemographic, resting lung function (forced expiratory volume in one second $\left[\mathrm{FEV}_{1}\right]$, forced vital capacity, synchronized vital capacity, IC), and quality of life data based on the Spanish version of Saint George's Respiratory Questionnaire (SGRQ) were obtained.

Results: Lung function results expressed as percentages of the predicted values were as follows: $\mathrm{FEV}_{1}, 49.04$ (standard deviation [SD]: 16.23); IC, 61.73 (SD: 15.42). The SGRQ mean total score was 47.5 (SD 17.98). The Spearman's Rho correlation between $\mathrm{FEV}_{1}$ and SGRQ was $\mathrm{r}=-0.36$ ( $95 \%$ confidence interval [CI]: -0.529 to -0.166 ), between IC and SGRQ was $\mathrm{r}=-0.329$ (95\% CI -0.502 to -0.131$)$, and between $\mathrm{FEV}_{1}$ and $\mathrm{IC}$ was $\mathrm{r}=0.561$.

Conclusions: Measurement of IC at rest could be used as a complementary functional exploration to forced spirometry in the monitorization of patients with COPD in the primary care setting. We found a poor correlation between IC and quality of life at the same level as in FEV .

Keywords: inspiratory capacity, primary care, quality of life, COPD

\section{Introduction}

Chronic obstructive pulmonary disease (COPD) is a chronic disease characterized by the loss of lung elasticity and airway narrowing resulting in airflow limitation. ${ }^{1,2}$ This progressive airflow limitation leads to chronic air trapping and hyperinflation, especially during activity or exercise. Hyperinflation causes mechanical disadvantages since it depresses the diaphragm and impairs intercostal muscle contractility. This impairment increases the work and metabolic expenditure associated with breathing and contributes to breathlessness. ${ }^{3}$

In the diagnosis of COPD, spirometry has been used as an objective measure to confirm its symptom-based clinical suspicion. The diagnostic criteria for COPD include a forced expiratory volume in one second/forced vital capacity ratio $\left(\mathrm{FEV}_{1} / \mathrm{FVC}\right)$ less than $70 \%$ of predicted value. ${ }^{1,2} \mathrm{FEV}_{1}$ in patients with COPD is used to grade the severity of airway obstruction ${ }^{4}$ and as the main predictor of both disease progression and mortality. However, $\mathrm{FEV}_{1}$ has limited application in clinically assessing patients. For example, patients with mild disease $\left(\mathrm{FEV}_{1}\right.$ higher than $80 \%$ of predicted value) or even patients with severe or very severe disease $\left(\mathrm{FEV}_{1}<60 \%\right.$ of predicted value $)$ show a poor correlation between their degree of bronchial obstruction and their clinical
Correspondence: Antonio Martín Pfizer Spain, Avenida de Europa 20B, Parque empresarial La Moraleja, 28108 Alcobendas, Madrid, Spain

Tel +344909628

Fax +34 9l 4909721

Email antonio.martin@pfizer.com 
situation. In these cases patients' quality of life (QoL) and survival can be conditioned by other factors. ${ }^{3}$

Regardless of the clinical usefulness of spirometry, clinical experience demonstrates that patients sharing very similar $\mathrm{FEV}_{1}$ values could show different blood gas values, clinical parameters, degrees of dyspnea, and very marked differences in QoL. These observations support the existence of other variables which may intervene in the pathogenesis or evolution of COPD. It is therefore necessary to consider other tests than $\mathrm{FEV}_{1}$ to asses the evolution of COPD patients. In these patients, other parameters such as inspiratory capacity (IC) may be more effective in detecting response to treatment, since they depend less on the degree of obstruction and mechanical compression associated with forced expiratory maneuvers. ${ }^{5}$ Previous studies have demonstrated the correlation between IC and the patient-centered outcomes of exercise tolerance and dyspnea. ${ }^{6}$

The aim of this study was to assess the usefulness of IC measurement in the clinical evaluation of COPD patients in the primary care setting. We looked for the relationship between the IC and $\mathrm{FEV}_{1}$ at rest and QoL in these patients.

\section{Methods}

This was a cross-sectional, observational study. Simple random patient sampling was carried out in COPD patients, following a table of random numbers, an initial list was selected after a search with keywords (COPD, chronic bronchitis, or chronic airflow obstruction) included in the informatic application TASS (National Health System application), and among those included in the integrated care process for COPD (COPD disease management program) of the Andalusian Health Council. Patients had to perform a pre- and postbronchodilator spirometry at the first visit in order to confirm their COPD diagnosis according to Spanish Thoracic Society (SEPAR) guidelines. ${ }^{2}$ The protocol was approved by ethic committee at the Puerta del Mar Hospital (Cádiz, Spain) and all patients provided written informed consent.

Patients were required to be older than 40 years and to have a spirometric diagnosis of COPD according to SEPAR guidelines. Exclusion criteria were: inability to perform the tests, ${ }^{2,7}$ previous history of asthma or comorbidities (neoplasm, other pulmonary and oropharyngeal obstructive disorders, tracheotomized patients, restrictive lung diseases, cardiac failure), and a history of exacerbation that required health care $^{1,4,8}$ within the 30 days prior to inclusion in the study.

The study population was recruited from eight primary care centers in Spain: La Laguna (Cádiz); El Carmen (El Puerto de
Santa María, Cádiz); Rodríguez Arias (San Fernando, Cádiz); Lucena (Córdoba); Dos Hermanas (Sevilla); Bollullos del Condado (Huelva); El Molino (Huelva); Fuente Blanca (Málaga). The inclusion period was six months.

After estimating a Pearson correlation coefficient of 0.29 for quantitative variables (coinciding with the value determined in similar studies in the primary care setting for $\left.\mathrm{FEV}_{1}\right),{ }^{9} P=0.05$, and a power of 0.80 for a bilateral hypothesis, the sample size was found to be 91 individuals (EPIDAT 3.0 software (Health Department of the Galician Government, Galicia, Spain). Sampling was applied to patients presenting criteria compatible with COPD and included in the TASS database and in the COPD integrated care program of the Andalusian health care authorities (Health Department of the Andalusian Government).

\section{Study variables}

Information was collected regarding anthropometric (weight, height) and sociodemographic (age, sex) parameters, smoking habit, QoL, severity (as defined by SEPAR guidelines, patients with $\mathrm{FEV}_{1}$ expressed as percentage compared to reference: mild $\mathrm{FEV}_{1}>80 \%$; moderate $\mathrm{FEV}_{1}$ 60\%-80\%; severe $\mathrm{FEV}_{1} 40 \%-60 \%$; very severe $\mathrm{FEV}_{1}<40 \%{ }^{2}$ ), specific treatment by therapeutic group in the preceding three months (short- and long-acting anticholinergics, short- and long-acting beta-adrenergics, inhaled/oral corticosteroids, and methylxanthines), oxygen therapy, adjustment of pharmacological treatment to disease severity according to SEPAR guidelines, ${ }^{2}$ and frequency of clinical exacerbations requiring attention in a primary care center, emergency room, or hospital admission.

Data were likewise collected on lung function as defined by $\mathrm{FEV}_{1}$ and IC. Testing met SEPAR recommendations. ${ }^{7}$ Reproducibility criteria were as defined by SEPAR guidelines. Spirometry was derived from flow-volume loops. After a period of tidal breathing, subjects were instructed to inhale to total lung capacity and then exhale as quickly and forcefully as possible to residual volume and then inhale back to tidal volume. Exhalation was continued until lungs were emptied completely with absence of further flow. $\mathrm{FEV}_{1}$ was derived from these loops. To measure IC, subjects completed four tidal breaths, inhaled to total lung capacity, and then exhaled slowly to residual volume. Exhalation was continued until lungs were emptied completely with absence of further flow. IC was calculated through the formula: IC = synchronized vital capacity (SVC) - expiratory reserve volume (ERV). Reference values used for the different expiratory variables were those obtained for the Spanish population..$^{10,11}$ 


\section{Measurement instruments}

Patients performed lung function testing with their usual pharmacological treatment. A Lilly-type Datospir 120D (Sibelmed, Barcelona, Spain) pneumotachometer spirometer was used.

To assess the COPD patients' QoL, the Spanish version of St. George's Respiratory Questionnaire (SGRQ), was used. ${ }^{12-14}$ This questionnaire included 50 items across three domains: symptoms, activity, and impact. The items corresponding to the symptoms domain refer to the frequency and severity of the respiratory symptoms. The activity domain addresses activity limitation due to breathlessness. The impact domain evaluates social functioning and psychological disturbances resulting from airways disease. The questionnaire items are presented in two ways: questions with up to five possible answers (only one can be chosen), or dichotomous-reply (yes/no) questions. The scores range from 0 to 100 , with lower scores indicating improvement and a deviation of four units or more were considered to be clinically meaningful.

\section{Data collection}

After being informed about the characteristics of the study and the confidentiality of the data, and once written consent was obtained, patients were scheduled for a visit in their corresponding primary care centers to assess their chronic airflow obstruction by spirometry. They were then instructed not to discontinue their usual pharmacological treatment.

In the scheduled visit, sociodemographic, anthropometric, and smoking data were collected. Patients were asked about their specific pharmacological treatment (if any). Both active drugs and daily doses were recorded. This was followed by self-administration of the SGRQ and posterior lung function test performance. Forced spirometry comprised the record of three correct maneuvers according to the standards for lung function test performance (SEPAR guideline recommendations which also met American Thoracic Society Task Force recommendations). Patients were finally instructed to performance a complete exhalation until SVC was obtained three times.

Data was collected through a paper case report form (CRF) specifically designed for this study. All data were introduced in a Microsoft Access database (Microsoft, Redmond, WA, USA) and processed with the SPSS statistical package (v. 12.0; SPSS Inc., Chicago, IL, USA).

\section{Statistical analysis}

Frequency tables for categorical variables were completed for our descriptive statistical study, and the mean, standard deviation (SD), and median for continuous variables were calculated. Calculation of the linear correlation coefficient between quantitative variables was based on the Spearman's Rho statistic for nonparametric tests. Confidence intervals were calculated for the correlation coefficient, using the Fisher transformation. The level of significance was established at $95 \%$.

\section{Results}

The results obtained from the random selection of patients (Figure 1) reveal inadequate quality of the diagnostic registries in the primary care clinical histories with mistakes in diagnosis in up to $30 \%$ of the patients. Because of the variability in the diagnoses registered in the medical history it was necessary to search the patient records with keywords that would allow greater sensitivity in the identification of eligible patients. The simple random sampling before the inclusion guarantees the same representation of all COPD patients according to disease severity. In the scheduled visit, the investigators confirmed that patients met the diagnostic criteria of COPD. Patients with recent exacerbations were excluded in order not to affect the results.

From a random selection of individuals, 93 patients were included from the population with COPD registered in the electronic case history database (Figure 1).

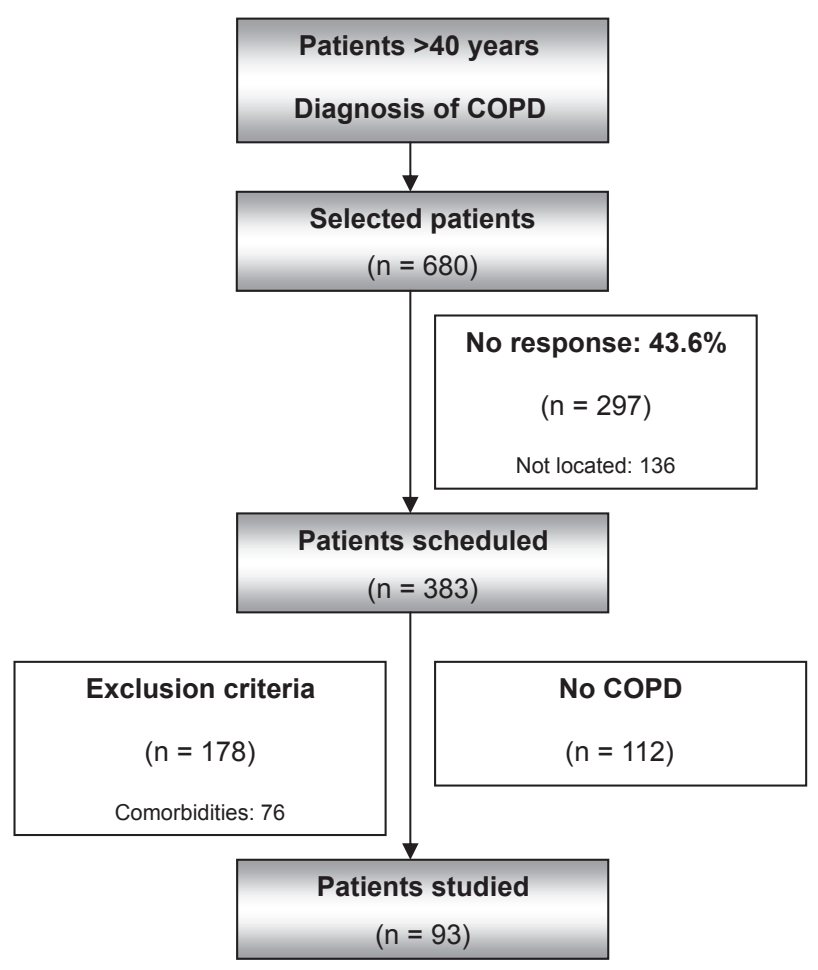

Figure I Study population. Total numbers with inclusion and exclusion criteria and nonresponse.

Abbreviation: COPD, chronic obstructive pulmonary disease. 
The mean age of the 93 participants was 68.4 years (SD 7.8), 96.8\% were male and 35.5\% were current smokers. Their COPD severity stage had the following distribution: mild, $5.4 \%$; moderate, $39.8 \%$; severe, $39.8 \%$; and very severe, $15 \%$. Pharmacological treatment followed guidelines ${ }^{1,2}$ in $59.1 \%$ of cases.

The characteristics of the study population showed most patients were male with conditions amenable to improvement such as smoking at the time of the study and treatment optimization according to disease severity.

Table 1 reports the bivariant analysis of lung function parameters $\left(\mathrm{FEV}_{1}, \mathrm{FEV}_{1} / \mathrm{FVC}\right.$, IC, forced expiratory flow [FEF] 25\%-50\%) and QoL, according to severity, gender, and therapeutic optimization. The mean total SGRQ score was 47.5 (SD 17.98). Table 2 shows the correlations between $\mathrm{FEV}_{1}$ and IC and total SGRQ score for each degree of severity. The correlation coefficient between $\mathrm{FEV}_{1}$ and SGRQ was -0.36 ( $95 \% \mathrm{CI}:-0.17$ to -0.53 ), and the correlation coefficient between IC and SGRQ was -0.33 (95\% CI: -0.13 to -0.50 ). Figure 2 presents the dispersion graphics and linear correlation values between IC and $\mathrm{FEV}_{1}$. The statistical correlation between $\mathrm{FEV}_{1}$ and IC was $\mathrm{r}=0.516$.

\section{Discussion}

Misdiagnosis between COPD and other respiratory diseases such as asthma could cause inadequate management of these diseases. There are a large number of patients who enter primary care practices with a prior respiratory diagnosis, or who have received respiratory medications without a clearly established diagnosis. ${ }^{15}$ Data from COPD patients in previous epidemiological studies in Spain has shown a high COPD prevalence $(9.1 \%$ in general population aged $>40$ years) and high rates of underdiagnosis ${ }^{16}(78.4 \%)$. The data demonstrates that most of these patients were incorrectly treated according to COPD guidelines. ${ }^{16}$ In this study, the percentage of misdiagnosis in our population was high: 29.2\% of COPD patients did not meet COPD spirometric diagnostic criteria. In order to efficiently identify which patients need further evaluation with spirometry, the general practitioner needs help to identify those patients who are most likely to have a fixed obstruction. ${ }^{15}$

Spirometry should be performed in all patients suspected of COPD to diagnose the disease and to assess disease severity. The spirometric definition of COPD includes the presence of a postbronchodilator $\mathrm{FEV}_{1} / \mathrm{FVC}<70 \%$ in order to confirm the presence of airflow limitation that is not fully reversible. ${ }^{17}$ Although these variables can be measured accurately and precisely in clinical research, they may be inconsistent in a primary care setting. This inconsistency may be related, at least in part, to incorrect interpretation of the spirometry results (only $47 \%$ of primary care physicians correctly interpreted spirometry results). ${ }^{18}$

As we have mentioned before, $\mathrm{FEV}_{1}$ has a poor correlation with clinical definition of COPD. ${ }^{3}$ In order to increase the correct diagnosis of patients with COPD, a possible alternative to $\mathrm{FEV}_{1}$ could be another parameter such as dyspnea, QoL, or IC. These parameters have established correlations with exercise tolerance and physical activity.

In COPD, hyperinflation or air trapping is the result of airway obstruction and the destruction of the lung parenchyma and its vasculature. In recent years, the dynamic hyperinflation in these patients has been recognized as a factor that triggers dyspnea and reduces exercise capacity. The degree of dynamic hyperinflation can be assessed by measuring reduction in IC. The measurement of IC in primary

Table I Bivariate analysis of the pulmonary function results and characteristics of the patients

\begin{tabular}{|c|c|c|c|c|c|}
\hline \multirow[t]{2}{*}{ Variables } & IC & FEV $_{1}$ & FVC & FEV, /FVC & FEF $25 \%-50 \%$ \\
\hline & Mean \% (SD) $P$ & Mean \% (SD) $P$ & Mean \% (SD) $P$ & Mean \% (SD) $P$ & Mean \% (SD) $P$ \\
\hline Sex & 0.896 & 0.161 & 0.492 & 0.148 & 0.153 \\
\hline Male & $62.88(4.59)$ & 62.00 (12.29) & $69.33(10.02)$ & $86.67(9.86)$ & 40.33 (10.69) \\
\hline Female & $61.69(15.66)$ & 48.61 (16.22) & $63.47(14.56)$ & 71.34 (I8.02) & $28.49(14.04)$ \\
\hline Severity & $<0.001$ & $<0.001$ & $<0.001$ & $<0.001$ & $<0.001$ \\
\hline Mild & 71.37 (16.09) & $82.60(2.79)$ & $90.00(8.09)$ & 87.40 (12.48) & $58.80(5.02)$ \\
\hline Moderate & 70.18 (I3.7I) & $61.67(6.32)$ & $72.38(9.11)$ & $80.25(15.20)$ & $38.50(8.77)$ \\
\hline Severe & 55.09 (II.35) & $40.46(5.69)$ & $57.57(9.76)$ & 68.03 (16.19) & $21.03(6.75)$ \\
\hline Very severe & $53.51(16.96)$ & $26.36(3.4 I)$ & $47.28(9.07)$ & 54.71 (14.95) & $|4.2|(5.92)$ \\
\hline
\end{tabular}

Note: Mean \%, mean percentage of theoretical value.

Abbreviations: IC, inspiratory capacity; $\mathrm{FEV}_{1}$, forced expiratory volume in one second; FVC, forced vital capacity; FEF $25 \%-50 \%$, forced expiratory flow $25 \%-50 \%$; SD, standard deviation. 
Table 2 Determination of linear correlation coefficients of IC and FEVI with the St. George's Respiratory Questionnaire and disease severity

\begin{tabular}{|c|c|c|c|c|}
\hline \multirow[t]{2}{*}{ Variables } & \multicolumn{2}{|l|}{ FEV } & \multicolumn{2}{|l|}{ IC } \\
\hline & Rho spearman (IC95\%) & $P$ & Rho spearman (IC95\%) & $P$ \\
\hline \multicolumn{5}{|l|}{ St. George's Respiratory } \\
\hline \multicolumn{5}{|l|}{ Questionnaire } \\
\hline Total score $(n=90)$ & $-0.36(-0.53 a-0.17)$ & $<0.00 I^{*}$ & $-0.33(-0.50 a-0.13)$ & $0.002 *$ \\
\hline Impact domain $(n=92)$ & $-0.28(-0.46 \mathrm{a}-0.08)$ & $0.007^{*}$ & $-0.31(-0.48 a-0.11)$ & $0.003 *$ \\
\hline Symptoms domain $(\mathrm{n}=92)$ & $-0.15(-0.34$ a 0.06$)$ & 0.16 & $-0.15(-0.35$ a 0.05$)$ & 0.142 \\
\hline Activity domain $(\mathrm{n}=92)$ & $-0.42(-0.57 \mathrm{a}-0.23)$ & $<0.001 *$ & $-0.32(-0.49 a-0.12)$ & $0.002^{*}$ \\
\hline \multicolumn{5}{|l|}{ Severity } \\
\hline Mild (n = 5) & $0.05(-0.87 \mathrm{a}-0.89)$ & 0.94 & $0.80(-0.28$ a 0.99$)$ & 0.10 \\
\hline Moderate $(n=37)$ & $-0.16(-0.46$ a 0.17$)$ & 0.34 & $-0.30(-0.567$ a 0.03$)$ & 0.08 \\
\hline Severe $(n=37)$ & $-0.38(-0.38 \mathrm{a}-0.06)$ & $0.022 *$ & $-0.40(-0.642$ a -0.09$)$ & $0.015^{*}$ \\
\hline Very severe $(n=14)$ & $0.07(-0.47$ a 0.58$)$ & 0.81 & $0.33(-0.243$ a 0.73$)$ & 0.27 \\
\hline
\end{tabular}

Note: *statistically significant.

Abbreviations: $\mathrm{Cl}$, confidence interval; IC, inspiratory capacity; $\mathrm{FEV}_{1}$, forced expiratory volume in one second.

care settings is very rarely performed and no references were found in this setting to date.

Hyperinflation in early COPD is not typically discerned by radiography but must be assessed objectively through lung volume testing (ie, body plethysmography).${ }^{19}$ Hyperinflation is discerned only in advanced COPD by radiography that shows an expanded chest, increased retrosternal air space, low and flat diaphragms, and decreased peripheral vascularity. Increased airway resistance, decreased lung elastic recoil and tethering properties, and premature airway closure results in an increased functional residual capacity (FRC). ${ }^{20}$

All COPD patients at rest show a variable degree of pulmonary hyperinflation, but IC reduction may have no consequences for gas exchange or may cause dyspnea. During exercise in COPD patients, the tidal volume doesn't increase properly because of reduced IC and results in incomplete emptying of the lungs during the expiration (air trapping). This becomes even more important when the intensity of physical exercise increases (and thus ventilation) giving rise to dynamic hyperinflation ${ }^{21}$ and worsening of air trapping, dyspnea, and exercise tolerance. ${ }^{22}$ The dynamic hyperinflation explains the limited exercise tolerance and breathing difficulties observed during daily life activities in COPD patients. ${ }^{23}$ The administration of bronchodilators in some cases may not improve $\mathrm{FEV}_{1}$ but they might improve air trapping and consequential dyspnea and exercise tolerance. $^{24,25}$

The results of the present study reveal the normal statistical distribution of IC values with dispersion of the values that allows correlations to be established with subjective measures of QoL perception. In our study, we observed a correlation between IC and SGRQ $(-0.33 ; 95 \%$ CI: -0.13 to -0.50$)$ showing that IC does affect the QoL of COPD patients. Similar correlation coefficients were observed between $\mathrm{FEV}_{1}$ and QoL and between IC and QoL as measured at rest by the SGRQ. Measurement of IC at rest may complement forced spirometry in the monitoring of patients with COPD in the primary care settings.

In contrast to parameters such as age, body mass index (BMI) or $\mathrm{FEV}_{1}$, a statistically significant and clinically relevant correlation is observed between the change in IC and dyspnea after exercise test (six-minute walk test). ${ }^{21}$ These observations suggest that the measurement of IC may constitute an objective indicator of dynamic hyperinflation and air trapping in COPD.

It seems reasonable to consider the usefulness of IC, with equivalence to $\mathrm{FEV}_{1}$ in its correlation to $\mathrm{QoL}$ perception, and with higher correlation to $\mathrm{FEV}_{1}$ in terms of dyspnea after exercise testing. ${ }^{26}$ However, there is insufficient evidence on the reliability, reproducibility, and interindividual variability of IC. Moreover, and unlike that in $\mathrm{FEV}_{1}$, the cut-off points or percentiles allowing classification of severity of the obstructive lung disease, its evolution over time, and the severity of air trapping or hyperinflation as a function of IC remain to be defined. While the results of the statistical correlation between $\mathrm{FEV}_{1}$ and IC for the population studied allow us to postulate our working hypotheses, further studies are required taking into account prognostic variables relating to COPD morbidity-mortality, in order to define the measurement of IC as a complement to $\mathrm{FEV}_{1}$, or even 

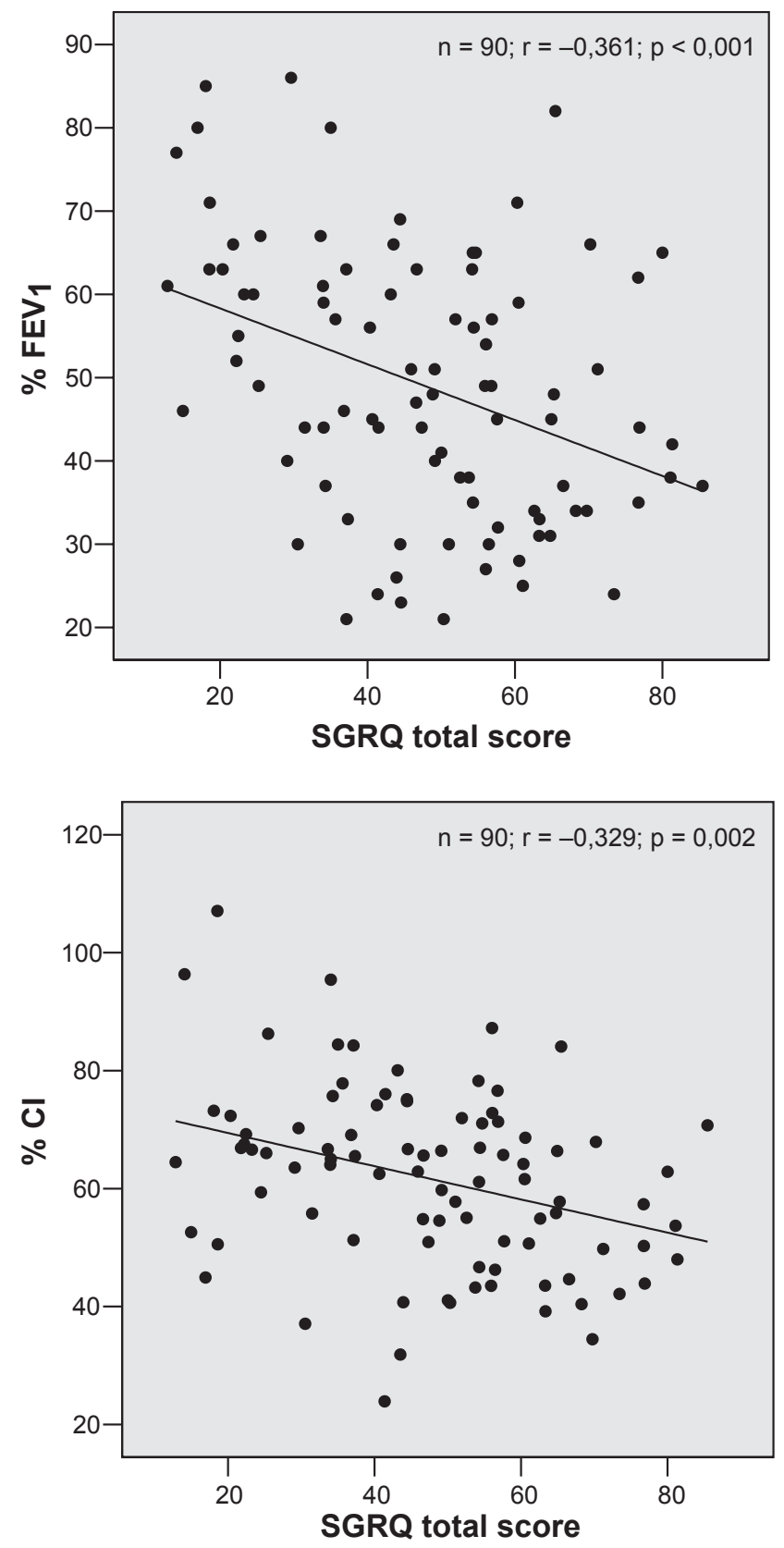

Figure 2 Dispersion and linear correlation values for the correlation between $\mathrm{FEV}_{1}$, IC, and the St. George's Respiratory Questionnaire score.

Abbreviations: IC, inspiratory capacity; $\mathrm{FEV}_{1}$, forced expiratory volume in one second.

as an alternative to forced spirometry in those cases where such exploration is difficult or not possible in the primary care setting.

We observed a relationship between the correlations of $\mathrm{FEV}_{1}$ and IC with the QoL reported by COPD patients. Taking in mind this correlation, we believe that the measurement of IC in primary care may be a useful complement to forced spirometry in the monitoring of COPD, particularly when forced spirometry is difficult or impossible to perform.

\section{Acknowledgments}

Members of the working group on respiratory disease Andalusian Society of Primary Care. To the medical departments of Pfizer SA and Boehringer Ingelheim by providing necessary resources for the study. To Dr Elena Gobartt for her contribution to initial study design and implementation.

\section{References}

1. Celli B, MacNee W. ATS/ERS Task Force. Standards for the diagnosis and treatment of patients with COPD. Eur Respir J. 2004;23:932-946.

2. Barberá JA, Peces Barba G, Agusti AG, et al. Sociedad Española de Neumologia y Cirugia Toracica (SEPAR). Guía clínica para el diagnóstico y tratamiento de la enfermedad pulmonar obstructiva crónica. Arch Bronconeumol. 2001;37:297-316.

3. Kilian K, Jones N. Respiratory muscles and dyspnea. Clin Chest Med. 1988;9:237-248.

4. Fabbri LM, Hurd SS. GOLD Scientific Committee. Global Strategy for the Diagnosis, Management and Prevention of COPD: 2003 update. Eur Respir J. 2003;22:1-2.

5. Izquierdo Alonso JL. Valoración funcional de la EPOC. Arch Bronconeumol. 2001;37(Suppl 2):2-8.

6. O'Donnell DE, Fluge T, Gerken F, et al. Effects of tiotropium on lung hyperinflation, dyspnea and exercise tolerance in COPD. Eur Respir J. 2004;117(Suppl 12A):49S-59S.

7. Grupo de trabajo de la SEPAR para la práctica de la espirometría en clínica. Recomendaciones SEPAR. Normativa para la práctica de la espirometría forzada. Arch Bronconeumol. 1989;25:132-141.

8. Anthonisen NR, Manfreda J, Warren CPW, Hershfield ES, Harding GKM, Nelson NA. Antibiotic therapy in exacerbations of chronic obstructive pulmonary disease. Ann Intern Med. 1987;106:196-204.

9. Fernandez Vargas AM, Bujalance Zafra MJ, Leiva Fernandez F, Martos Crespo F, García Ruíz A, Prados Torres D. Correlación entre medidas de salud subjetivas y objetivas en pacientes con enfermedad pulmonar obstructiva crónica (EPOC). Atención Primaria. 2001;28:579-589.

10. Roca J, Sanchis J, Augusti-Vidal A, et al. Spirometric reference values from a Mediterranean population. Bull Eur Physiopathol Respir. $1986 ; 22: 217-224$

11. Air pollution and chronic or repeated respiratory diseases. II. Results and discussion. Bull Eur Physiopathol Respir. 1982;18:101-102.

12. Ferrer M, Alonso J, Prieto L, et al. Validity and reliability of the St. George's Respiratory Questionnaire after adaptation to a different language and culture: the Spanish example. Eur Respir J. 1996;9: $1160-1166$.

13. Ferrer M, Alonso J, Morera J, et al; Chronic obstructive pulmonary disease stage and health-related quality of life. The Quality of Life of Chronic Obstructive Pulmonary Disease Study Group. Ann Intern Med. 1997;127:1072-1079.

14. Badia X, Salamero M, Alonso J. La Medida de la Salud. Guía de escalas de medición en español. Cuestionario Respiratorio St. George. Edimac edit. Barcelona, Spain: Press Line; 1999. p. 175-181.

15. Tinkelman DG, Price DB, Nordyke RJ, et al. Symptom-based questionnaire for fifferentiating COPD and asthma. Respiration. 2006;73: 296-305.

16. Peña VS, Miravitlles M, Gabriel R, et al; Geographic variations in prevalence and underdiagnosis of COPD: results of the IBERPOC multicentre epidemiological study. Chest. 2000;118(4):981-989.

17. Rabe KF, Beghé B, Luppi F, Fabbri LM. Update in chronic obstructive pulmonary disease 2006. Am J Respir Crit Care Med. 2007;175: 1222-1232.

18. Eaton T, Withy S, Garrett JE, Mercer J, Whitlock RM, Rea HH. Spirometry in primary care practice: the importance of quality assurance and the impact of spirometry workshops. Chest. 1999;116(2):416-423. 
19. O’Donnell DE, Revill SM, Webb KA. Dynamic hyperinflation and exercise intolerance in chronic obstructive pulmonary disease. Am J Respir Crit Care Med. 2001;164:770-777.

20. Rothpearl A, Varma AO, Goodman K. Radiographic measures of hyperinflation in clinical emphysema. Discrimination of patients from controls and relationship to physiologic and mechanical lung function. Chest. 1988;94:907-913.

21. Marín Trigo JM. Manifestaciones clínica: la disnea y su importancia en el paciente con EPOC. Arch Bronconeumol. 2001;37(Suppl 2):8-13.

22. Cooper $\mathrm{CB}$. The connection between chronic obstructive pulmonary disease symptoms and hyperinflation and its impact on exercise and function. Am J Med. 2006;119(10A):S21-S31.

23. Marín JM, Carrizo SJ, Gascón M, Sánchez A, Gallego B, Celli BR. Inspiratory capacity, dynamic hyperinflation, breathlessness, and exercise performance during the 6-minute-walk test in chronic obstructive pulmonary disease. Am J Respir Crit Care Med. 2001;163:1395-1399.
24. Belman MG, Botnick WC, Shin JW. Inhaled bronchodilators reduce dynamic hyperinflation during exercise in patients with chronic obstructive pulmonary disease. Am J Respir Crit Care Med. 1996;153:967-975.

25. Celli B, ZuWallack R, Wang S, Kesten S. Improvement in resting inspiratory capacity and hyperinflation with tiotropium in COPD patients with increased static lung volumes. Chest. 2003;124: 1743-1748

26. Pitta F, Takaki MY, de Oliveira NH, et al. Relationship between pulmonary function and physical activity in daily life in patients with COPD. Respir Med. 2008;102:1203-1207.
International Journal of General Medicine

\section{Publish your work in this journal}

The International Journal of General Medicine is an international, peer-reviewed open-access journal that focuses on general and internal medicine, pathogenesis, epidemiology, diagnosis, monitoring and treatment protocols. The journal is characterized by the rapid reporting of reviews, original research and clinical studies across all disease areas.

\section{Dovepress}

A key focus is the elucidation of disease processes and management protocols resulting in improved outcomes for the patient.The manuscript management system is completely online and includes a very quick and fair peer-review system. Visit http://www.dovepress.com/ testimonials.php to read real quotes from published authors.

Submit your manuscript here: http://www.dovepress.com/international-journal-of-general-medicine-journal 